\title{
Hemoglobinopatías en comunidades de raza negra de los ríos Cayapas y Onzoles, cantón Eloy Alfaro, provincia de Esmeraldas, Ecuador
}

\author{
Angel Guevara, Martha Chico, Manuel Calvopiña, Ronald H. Guderian
}

\begin{abstract}
Resumen
Se analizaron muestras de sangre de 1.364 individuos de raza negra pura, habitantes de la ribera de los ríos Cayapas y Onzoles, norte de la provincia de Esmeraldas, para hemoglobinopatías mediante electrofóresis de hemoglobina sobre acetato de celulosa y agar de citrato. El 75,7\% (1.032) de la población estudiada presenta hemoglobina normal y $24,3 \%$ (332) tiene variantes de hemoglobina. La variante más frecuente fue $\mathrm{Hb}$.AS con $84,9 \%$ (282), seguida de Hb.AF 9,6\% (32), Hb.SS 2,7\% (9), Hb.FS 2,4\% (8) y Hb.FF $0,3 \%$ (1). La prevalencia más elevada se detectó en los grupos de edad jóvenes, 0-9 años $(31,8 \%)$ y $10-19$ años $(25,8 \%)$. Los hombres presentaron mayor prevalencia que las mujeres en los dos ríos estudiados y existen áreas geográficas características con alta prevalencia en el río Cayapas, Trinidad (45,5\%) y Santa María (37,9\%), mientras que en el río Onzoles la prevalencia es más uniforme. Se discuten los hallazgos encontrados, principalmente, la prevalencia del genotipo $\mathrm{Hb}$.SS $(2,7 \%)$ y de un caso $(0,3 \%)$ con características de Hb.FF que merecen ser estudiados con más detalle.
\end{abstract}

\section{Haemoglobinopathies in black communities living along the banks of the rivers Cayapas and Onzoles, in the Eloy Alfaro canton, in the province of Esmeraldas, Ecuador.}

Blood samples from 1,364 individuals of Black race, inhabitants of the area bordering the Cayapas and Onzoles rivers, located in the northwest corner of the Esmeraldas' province, were analysed for haemoglobinopathies by means of haemoglobin electrophoresis using cellulose acetate and citrate agar plates. Of the studied population, $75.7 \%(1,032)$ were found to have normal haemoglobin and $24.3 \%$ (332) had some homoglobin variant. The most frequent variant was $\mathrm{Hb}$.AS with $84.9 \%$ (282), followed by Hb.AF $9.6 \%$ (32), Hb.SS $2.7 \%(9)$, Hb.FS $2.4 \%$ (8) and Hb.FF $0.3 \%$ (1). The highest prevalence of haemoglobinopathies $(31.8 \%)$ was detected in young people, $0-9$ years of age, and in those aged $10-19$ (25.8\%). A higher prevalence was found in males than females throughout the two river areas studied. On the Cayapas river, certain geographical areas were found to have a high prevalence of haemoglobinopathies such as in Trinidad (45.5\%) and Santa María (37.9\%), while on the Onzoles river the prevalence was more uniform. These findings are discussed, particularly the prevalence of the genotype $\mathrm{Hb}$.SS $(2.7 \%)$ and the one case $(0.3 \%)$ with $\mathrm{Hb}$.FF electrophoretic characteristics which requires more detailed study.

Departamento de Investigaciones Clínicas Servicios comunitarios, Hospital Vozandes, Ecuador. Casilla 17-17-691-Quito Ecuador.

Recibido para su publicación: 22 de abril de 1998 - Aprobado para su publicación: 30 de abril de 1998. 
Las variantes de hemoglobina características de la raza negra se presentan con una distribución casi universal. En particular, en países de América tropical con poblaciones de raza negra como Brasil, Cuba, Costa Rica, Colombia, Surinam, Guatemala y Haití se han descrito múltiples variantes en el patrón electroforético y genotípico de hemoglobinopatías heredadas por la migración africana producida a partir de 1518 (1).

En Ecuador, al igual que en los demás países de la América tropical, la población es muy heterogénea y está constituida en un gran porcentaje por mestizos y grupos raciales puros de indígenas y negros. La población negra, en Ecuador, tiene áreas geográficas muy definidas como la provincia de Esmeraldas en la costa y los valles interandinos cálidos como el Chota en la provincia de Imbabura y el Catamayo en la provincia de Loja (2). En la provincia de Esmeraldas, se estima que $60 \%$ de la población es negra. Dada la importancia clínica, antropológica y social del conocimiento de la distribución y prevalencia de hemoglobinopatías en grupos poblacionales específicos y debido a que no existen referencias anteriores, $s e$ estudiaron poblaciones negras puras que habitan en las riberas de los ríos Cayapas y Onzoles de la provincia de Esmeraldas.

En este artículo se informa la prevalencia y la distribución de las variantes de hemoglobinas detectadas en el área noreste de la provincia de Esmeraldas.

\section{Materiales y métodos}

Se estudiaron 1.364 individuos de raza negra pura, distribuidos en 13 localidades principales a lo largo de los ríos Cayapas y Onzones en la provincia de Esmeraldas (figura 1). La búsqueda de individuos para el estudio fue al azar y se evitó la inclusión de miembros familiares. En todos los individuos, se recolectaron muestras de sangre venosa anticoagulada con EDTA (acido-etilene-dinitroilotetra-acético) y se preservaron a $4^{\circ} \mathrm{C}$ hasta su procesamiento.

La investigación de variantes de hemoglobina se realizó mediante electrofóresis sobre acetato de celulosa (Titan III Cellulose Acetato Plates, Helena Laboratories, Texas, EUA) en solución tampón tris- barbital-sodium-barbital pH 8,8 (Gelman Instruments Co., Michigan, EUA). Para diferenciar la hemoglobina $S$ de la $C$ y la $D$ que migran juntas en acetato de celulosa, se realizaron fraccionamientos de hemoglobinas específicas mediante electrofóresis sobre agar de citrato ( Titan IV Plates, Helena Laboratories, Texas, EUA) en una solución tampón de citrato $0,05 \mathrm{M}$ a pH 6,2.

\section{Resultados}

1.364 individuos de la raza negra de 13 diferentes comunidades de los ríos Cayapas y Onzoles fueron examinados para variantes de hemoglobina. De los examinados, $1.043(76,6 \%)$ habitan en la rivera del río Cayapas y $321(23,4 \%)$ en el río Onzoles. La distribución según la edad de los examinados se presenta en el cuadro 1. La mayoría (792, $58,1 \%$ ) de los individuos estudiados tenía menos de 20 años de edad.

EI $75,7 \%$ (1.032) de la población estudiada presentó un patrón electroforético de hemoglobina normal. En $24,3 \%$ (332) se detectaron variantes de hemoglobina. La prevalencia de los casos positivos totales para variantes de hemoglobina, por cada una de las áreas examinadas y de acuerdo con la edad, se detalla en el cuadro 2. El mayor porcentaje, $31,8 \%$, de las variantes fenotipicas se encontró en el grupo de edad de 0 a 9 años, seguido por el grupo de 10 a 19 años con $25,8 \%$. A partir de este grupo, se apreció una disminución progresiva hasta el grupo de edad de 30 a 39 años, despúes del cual los valores se mantenían relativamente constantes. En general, los hombres demostraron una mayor prevalencia $(26,5 \%)$ que las mujeres $(22,2 \%)$, tanto en el río Cayapas como en el río Onzoles. Sin embargo, la prevalencia total encontrada en el río Cayapas $(25,0 \%)$ fue mayor a la detectada en el río Onzoles $(22,0 \%)$. Curiosamente, en el río Cayapas, el grupo de edad de 0 a 9 años tenía la prevalencia más alta $(36,1 \%)$ mientras que en el río Onzoles, la mayor prevalencia $(31,2 \%)$ se encontró en individuos mayores de 60 años. En el río Onzoles, los hombres a partir de los 40 años tenían una prevalencia superior de $40 \%$ para hemoglobinopatías.

La distribución de la prevalencia de hemoglobinopatías en relación con las áreas geográficas estudiadas y al sexo se encuentra en el cuadro 3. 


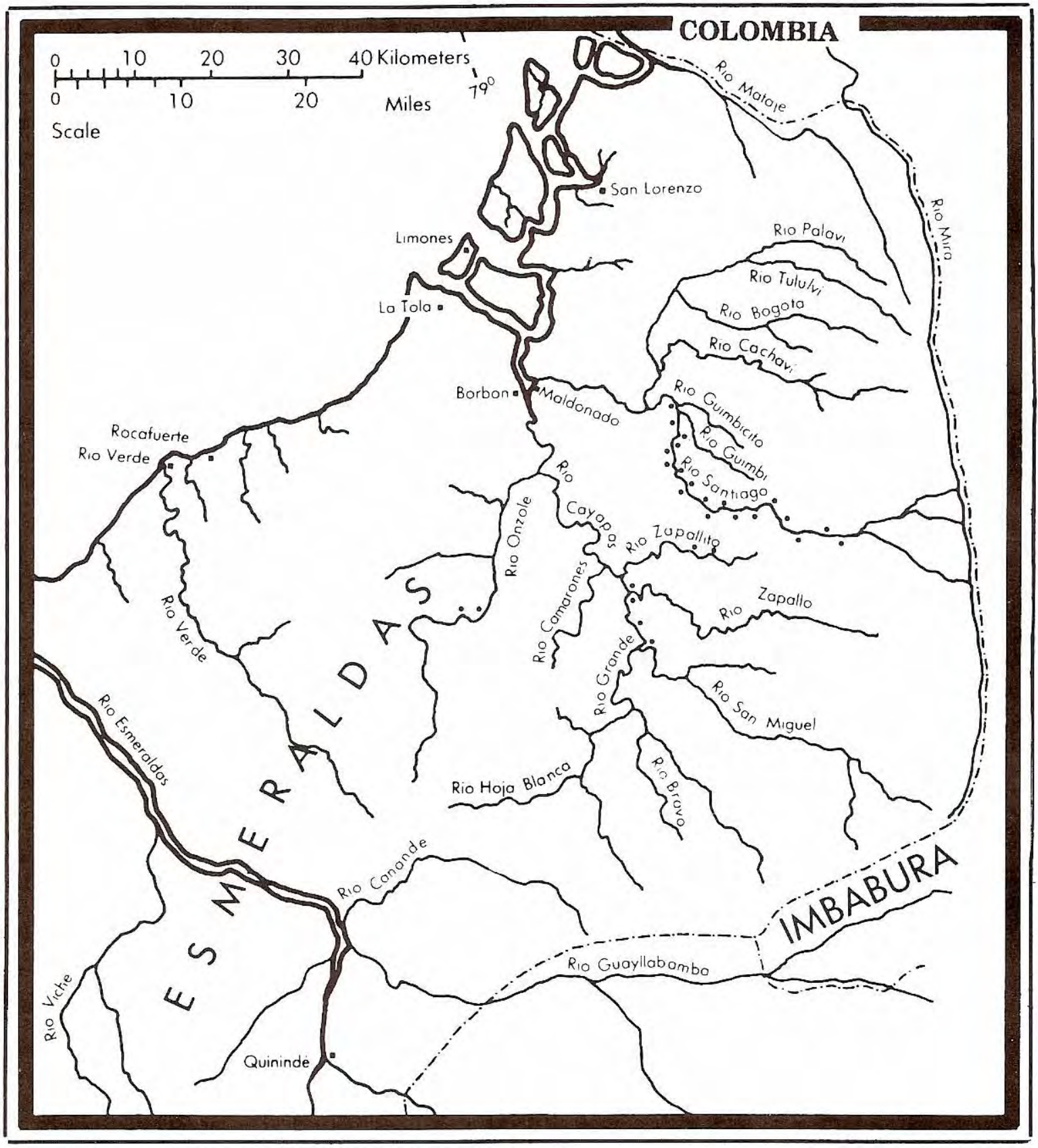

En el río Cayapas, las comunidades de Trinidad $(45,5 \%)$ y Santa María $(37,9 \%)$ tenían la mayor prevalencia para hemoglobinopatías. En el río Onzoles, las comunidades de Santo Domingo $(28,9 \%)$ y Colón $(28,0 \%)$ son las de mayor prevalencia. Las prevalencias más bajas se encon- traron en las comunidades de Borbón $(12,4 \%)$ y San Miguel $(13,1 \%)$ en el río Cayapas y Anchayacu $(16,0 \%)$ en el río Onzoles.

Las demás comunidades tuvieron aproximadamente la misma incidencia, $26,5 \% \pm 3,8 \%$. En 
Cuadro 1. Distribución de acuerdo a la edad y sexo, de los examinados para hemoglobinopatías, en los ríos Cayapas y Onzoles, provincia de Esmeraldas, Ecuador.

\begin{tabular}{crrrrrrrr}
\hline \multirow{2}{*}{$\begin{array}{c}\text { Edad } \\
\text { (años) }\end{array}$} & \multicolumn{2}{c}{ Examinado } & \multicolumn{3}{c}{ Río Cayapas } & \multicolumn{3}{c}{ Río Onzoles } \\
\cline { 3 - 8 } & $\mathbf{n}$ & $\%$ & Total & Hombre & Mujer & Total & Hombre & Mujer \\
\hline $0-9$ & 287 & 21,1 & 182 & 110 & 72 & 105 & 55 & 50 \\
$10-19$ & 505 & 37,0 & 420 & 226 & 194 & 85 & 43 & 42 \\
$20-29$ & 202 & 14,8 & 156 & 75 & 81 & 46 & 11 & 35 \\
$30-39$ & 130 & 9,5 & 104 & 38 & 66 & 26 & 8 & 18 \\
$40-49$ & 113 & 8,3 & 84 & 36 & 48 & 29 & 7 & 22 \\
$50-59$ & 78 & 5,7 & 64 & 32 & 32 & 14 & 5 & 9 \\
$60+$ & 49 & 3,6 & 33 & 14 & 19 & 16 & 7 & 9 \\
\hline
\end{tabular}

Cuadro 2. Prevalencia (\%) de hemoglobinopatías de acuerdo a la edad y sexo en los ríos Cayapas y Onzoles, provincia de Esmeraldas, Ecuador.

\begin{tabular}{cccccccccc}
\hline \multirow{2}{*}{$\begin{array}{c}\text { Edad } \\
\text { (años) }\end{array}$} & \multicolumn{2}{c}{ Total Examinados } & \multicolumn{2}{c}{ Río Cayapas } & \multicolumn{2}{c}{ Río Onzoles } \\
\cline { 2 - 9 } & Total & Hombre & Mujer & Total & Hombre Mujer & \multicolumn{2}{c}{ Total } & Hombre Mujer \\
\hline $0-9$ & 31,8 & 33,8 & 24,2 & 36,1 & 38,7 & 32,3 & 26,0 & 25,9 & 26,0 \\
$10-19$ & 25,8 & 23,8 & 29,4 & 26,8 & 25,0 & 29,3 & 21,7 & 19,0 & 24,4 \\
$20-29$ & 20,7 & 26,4 & 16,4 & 21,7 & 27,4 & 16,4 & 17,4 & 16,7 & 17,6 \\
$30-39$ & 19,1 & 26,1 & 15,6 & 19,8 & 26,5 & 16,4 & 15,4 & 22,2 & 11,8 \\
$40-49$ & 20,0 & 24,4 & 17,3 & 18,1 & 20,0 & 16,7 & 24,1 & 42,9 & 18,2 \\
$50-59$ & 17,3 & 21,1 & 14,0 & 17,7 & 16,1 & 19,4 & 14,3 & 40,0 & 00,0 \\
$60+$ & 21,2 & 22,7 & 20,0 & 15,2 & 14,3 & 15,8 & 31,2 & 42,9 & 22,2 \\
\hline
\end{tabular}

Cuadro 3. Distribución de acuerdo al sexo y sitios geográficos de los casos positivos para hemoglobinopatías en los ríos cayapas y Onzoles, provincia de esmeraldas, Ecuador

\begin{tabular}{|c|c|c|c|c|c|c|c|c|c|}
\hline \multirow{2}{*}{$\begin{array}{c}\text { Sitio } \\
\text { Geográfico }\end{array}$} & \multicolumn{3}{|c|}{ Total } & \multicolumn{3}{|c|}{ Hombre } & \multicolumn{3}{|c|}{ Mujer } \\
\hline & Exam. & Pos. & $\%$ & Exam. & Pos. & $\%$ & Exam. & Pos. & $\%$ \\
\hline \multicolumn{10}{|l|}{ Río Cayapas } \\
\hline Borbón & 314 & 39 & 12,4 & 145 & 25 & 17,2 & 168 & 14 & 8,3 \\
\hline Playa Grande & 44 & 10 & 22,7 & 21 & 5 & 23,8 & 23 & 5 & 21,7 \\
\hline Herradura & 14 & 4 & 28,5 & 6 & 2 & 33,3 & 8 & 2 & 25,0 \\
\hline San José & 87 & 23 & 26,4 & 43 & 11 & 25,6 & 44 & 12 & 27,2 \\
\hline Sta. Maria & 137 & 52 & 37,9 & 79 & 32 & 40,5 & 58 & 20 & 34,5 \\
\hline Trinidad & 79 & 36 & 45,5 & 47 & 19 & 40,4 & 32 & 17 & 53,1 \\
\hline Telembí & 93 & 26 & 27,9 & 55 & 15 & 27,3 & 38 & 11 & 28,9 \\
\hline Zapallo Grande & 239 & 60 & 25,1 & 123 & 31 & 25,2 & 116 & 29 & 25,0 \\
\hline San Miguel & 38 & 5 & 13,1 & 22 & 2 & 9,1 & 16 & 3 & 18,8 \\
\hline \multicolumn{10}{|l|}{ Río Onzoles } \\
\hline Achayacu & 50 & 8 & 16,0 & 24 & 5 & 20,8 & 26 & 3 & 11,5 \\
\hline San Francisco & 118 & 26 & 22,0 & 46 & 12 & 26,1 & 72 & 14 & 19,4 \\
\hline Sto. Domingo & 76 & 22 & 28,9 & 37 & 8 & 21,6 & 39 & 14 & 35,9 \\
\hline Colón & 76 & 21 & 27,6 & 32 & 13 & 40,6 & 44 & 8 & 18,2 \\
\hline Total & 1.364 & 332 & 24,3 & 680 & 180 & 26,5 & 684 & 152 & 22,2 \\
\hline
\end{tabular}


relación al sexo, los hombres presentaron prevalencias más elevadas en Trinidad $(40,4 \%)$ y Santa María (40,5\%) en el río Cayapas y en Colón $(40,6 \%)$ en el río Onzoles. La prevalencia más alta en las mujeres fue en Trinidad $(53,1 \%)$ en el río Cayapas y en Santo Domingo $(35,9 \%)$ en el río Onzoles.

Las distribuciones de los diferentes genotipos de acuerdo con la edad se encuentra en el cuadro 4. El genotipo más frecuente fue AS y representa el $84,9 \%$ (282) de todas las hemoglobinopatías detectadas; en orden descendente, se detectaron también los siguientes genotipos: AS fue uniforme para todas las edades. El genotipo SS se encontró solamente en niños con edades menores de 12 años. El genotipo AF presentó una frecuencia elevada $(9,6 \%)$ y se le encontró en todas las edades. La presencia del genotipo FF en un hombre de 43 años de edad es muy rara. No hubo una diferencia significativa entre hombres y mujeres.

Los genotipos en relación con los diferentes sitios geográficos se encuentra en el cuadro 5 . Todas las localidades estudiadas presentan el genotipo AS, siendo la mayor frecuencia de este genotipo en Trinidad, 40,5\%(32); en la misma comunidad, se encontró la frecuencia más elevada del genotipo SS $(5,1 \%)$. El genotipo AF se halla en 9 de las 13 localidades con su frecuencia más alta en San Francisco, 7,6\% (9), del río Onzoles.

\section{Discusión}

La prevalencia de hemoglobinopatías detectada en

Cuadro 4. Distribución de hemoglobinopatías de acuerdo a la edad en la raza negra en los ríos Cayapas y Onzoles, provincia de Esmeraldas, Ecuador.

\begin{tabular}{|c|c|c|c|c|c|c|c|c|c|c|c|c|}
\hline \multirow{2}{*}{$\begin{array}{l}\text { Edad } \\
\text { (años) }\end{array}$} & \multicolumn{2}{|c|}{ Total } & \multicolumn{2}{|c|}{ AS } & \multicolumn{2}{|c|}{ SS } & \multicolumn{2}{|c|}{ AF } & \multicolumn{2}{|c|}{ FS } & \multicolumn{2}{|c|}{ FF } \\
\hline & $n$ & $\%$ & $\mathrm{n}$ & $\%$ & $n$ & $\%$ & $\mathrm{n}$ & $\%$ & $n$ & $\%$ & $\mathrm{n}$ & $\%$ \\
\hline $0-9$ & 85 & 25,9 & 69 & 81,1 & 6 & 7,1 & 9 & 10,6 & 1 & 1,2 & 0 & 0,0 \\
\hline $10-19$ & 119 & 35,8 & 97 & 81,5 & 3 & 2,5 & 14 & 11,8 & 5 & 4,2 & 0 & 0,0 \\
\hline $20-29$ & 44 & 13,3 & 39 & 88,6 & 0 & 0,0 & 4 & 9,1 & 1 & 2,3 & 0 & 0,0 \\
\hline $30-39$ & 28 & 8,4 & 26 & 92,9 & 0 & 0,0 & 2 & 7,1 & 0 & 0,0 & 0 & 0,0 \\
\hline $40-49$ & 27 & 8,1 & 25 & 92,6 & 0 & 0,0 & 1 & 3,7 & 0 & 0,0 & 1 & 0,0 \\
\hline $50-59$ & 17 & 5,1 & 16 & 94,1 & 0 & 0,0 & 1 & 5,9 & 0 & 0,0 & 0 & 0,0 \\
\hline $60+$ & 12 & 3,6 & 10 & 83,4 & 0 & 0,0 & 1 & 8,3 & 1 & 8,3 & 0 & 0,0 \\
\hline Total & 332 & 100,0 & 282 & 84,9 & 9 & 2,7 & 32 & 9,6 & 8 & 2,4 & 1 & 0,3 \\
\hline
\end{tabular}

Cuadro 5. Variantes de hemoglobinas detectadas en las principales poblaciones de raza negra en los ríos Cayapas y Onzoles, Provincia de Esmeraldas, Ecuador.

\begin{tabular}{|c|c|c|c|c|c|c|c|c|c|c|}
\hline \multirow{3}{*}{$\begin{array}{c}\text { Sitio } \\
\text { Geográfico }\end{array}$} & \multicolumn{10}{|c|}{ Prevalencia (\%) Variantes Genotipos } \\
\hline & \multicolumn{2}{|c|}{ AS } & \multicolumn{2}{|c|}{ SS } & \multicolumn{2}{|c|}{ AF } & \multicolumn{2}{|c|}{ FS } & \multicolumn{2}{|c|}{ FF } \\
\hline & $\mathbf{n}$ & $\%$ & $\mathbf{n}$ & $\%$ & $\mathrm{n}$ & $\%$ & $\mathbf{n}$ & $\%$ & $\mathbf{n}$ & $\%$ \\
\hline \multicolumn{11}{|l|}{ Río Cayapas } \\
\hline Borbón & 39 & 12,4 & 0 & 0,0 & 0 & 0,0 & 0 & 0,0 & 0 & 0,0 \\
\hline Playa Grande & 8 & 18,1 & 1 & 2,3 & 1 & 2,3 & 0 & 0,0 & 0 & 0,0 \\
\hline Herraura & 4 & 28,5 & 0 & 0,0 & 0 & 0,0 & 0 & 0,0 & 0 & 0,0 \\
\hline San José & 20 & 23,0 & 0 & 0,0 & 2 & 2,2 & 1 & 1,2 & 0 & 0,0 \\
\hline Sta. María & 43 & 26,8 & 1 & 0,8 & 6 & 4,4 & 2 & 1,5 & 0 & 0,0 \\
\hline Trinidad & 32 & 40,5 & 4 & 5,1 & 0 & 0,0 & 0 & 0,0 & 0 & 0,0 \\
\hline Telembí & 23 & 24,7 & 0 & 0,0 & 2 & 2,2 & 1 & 1,1 & 0 & 0,0 \\
\hline Zapallo Grande & 57 & 23,8 & 0 & 0,0 & 3 & 1,3 & 0 & 0,0 & 0 & 0,0 \\
\hline San Miguel & 5 & 13,1 & 0 & 0,0 & 0 & 0,0 & 0 & 0,0 & 0 & 0,0 \\
\hline \multicolumn{11}{|l|}{ Río Onzoles } \\
\hline Anchayacu & 4 & 8,0 & 0 & 0,0 & 2 & 4,0 & 1 & 2,0 & 1 & 2,0 \\
\hline San Francisco & 16 & 13,6 & 1 & 0,8 & 9 & 7,6 & 0 & 0,0 & 0 & 0,0 \\
\hline Sto. Domingo & 18 & 23,7 & 0 & 0,0 & 2 & 2,6 & 2 & 2,6 & 0 & 0,0 \\
\hline Colón & 13 & 17,3 & 2 & 2,7 & 5 & 6,7 & 1 & 1,3 & 0 & 0,0 \\
\hline
\end{tabular}


Ecuador (24,3\% ) es una de las más altas que se han publicado en América Latina. Las hemoglobinas AS y AC se han detectado en Brasil con frecuencias, en negros, desde $4,8 \%$ hasta $15,0 \%$ según la región estudiada (3); en Cuba, se refieren frecuencias de 15,0\% (4); en Costa Rica, de 10,6\% (5); en Surinam, en varios estudios realizados, las frecuencias varían entre $11,5 \%$ (8). En Colombia, se han comunicado frecuencias entre $7,7 \%$ y $14,7 \%$, pero, únicamente con respecto a la Hb.AS (9). En Guatemala, la frecuencia de hemoglobinopatías es de $18,3 \%$, sin detectarse hemolobina C (10). Hay que señalar que la región estudiada es fronteriza con regiones de Colombia con alta prevalencia de hemoglobinopatías y las poblaciones analizadas están evolutivamente relacionadas; este hecho sumado a la alta endemicidad de la región para malaria pueden explicar la prevalencia de hemoglobinopatías en estas áreas de Ecuador.

Aparentemente, el gen de la hemoglobina S apareció, por lo menos, en tres ocasiones separadas en regiones del Africa: Senegal, Benin y República Africana Central (11). Así, la prevalencia del gen en ciertos países africanos es relativamente alta; por ejemplo, en Nigeria hay una prevalencia promedio de $20 \%$ y en algunas tribus se encontraron prevalencias superiores a $45 \%$ (12).

Se ha sugerido que la razón para la persistencia del gen en zonas donde se esperaría su desaparición por la alta mortalidad asociada con drepanocitosis, es que confiere una protección contra el paludismo. Las características físicas de los eritrocitos que contienen hemoglobina $S$ los hacen menos susceptibles a la parasitización por Plasmodium falciparun, lo que constituye un factor de protección para aquellos individuos heterocigotos para hemoglobina S (13). En Africa $y$ en otras regiones del mundo, existe una resistencia innata contra la infección malárica por $P$. falciparum en portadores heterocigotos de glóbulos rojos en forma de hoz (sickle cell) (14). Estas observaciones se confirman también en Ecuador, donde se encontró parasitemia con menor frecuencia en individuos que tenían variantes anómalas de hemoglobina (15).

También hay evidencia que Hb.C y Hb.F pueden perjudicar el crecimiento del parásito en el glóbulo rojo $(16,17)$; por tanto, la alta frecuencia de Hb.F encontrada en los morenos del Ecuador podría contribuir a la baja prevalencia de infecciones maláricas por $P$. falciparum encontrada en dicha raza.

La alta prevalencia del genotipo AS, detectada en este estudio, presenta riesgo si los individuos que lo poseen, migran a poblaciones de gran altura, donde la tensión de oxígeno disminuye lo cual favorece al fenómeno de drepanocitosis (células rojas en forma de hoz). Además, existen otras situaciones donde las personas con genotipos AS pueden desarrollar cuadros de drepanocitosis, así: viajes aéreos en condiciones de despresurización, hipoxia bajo anestesia y neumonías severas. Igualmente, la comunidad de Trinidad tiene un alto porcentaje del genotipo SS y se justifica un estudio clínico-hematológico más completo para tratar de explicar este hecho. Es importante recalcar que el genotipo SS fue encontrado solamente en niños y que constituye el primer informe sobre esta hemoglobinopatía en este grupo de edad en Ecuador.

Finalmente, en el río Onzoles, se encontraron genotipos que, por la migración electroforética en acetato de celulosa, asemejan a FF; sin embargo, mediante electrofóresis en agar de citrato no se confirma este genotipo y no coincide con los genotipos conocidos; por esta razón, es necesario implementar estudios más profundos para caracterizar este tipo de hemoglobina.

\section{Agradecimientos}

A los promotores de salud, señor Gabriel Ayovi y señor Eliseo Medina por la ayuda en la recolección de las muestras de las comunidades de los ríos Cayapas y Onzoles.

\section{Referencias}

1. Mornes M. La mezcla de razas en la historia de América Latina. Buenos Aires: Ed. Paidos; 1969. p. 28-30.

2. Banco Central del Ecuador. Atlas del mundo ECUADOR. París: Les Editions J.A.; 1982. p. 44.

3. Salzano FM. Genética de las poblaciones humanas brasileiras. En homenaje a J. Comas. Volumen II. México: Libro de México; 1965. p. 253-3-18. 
4. Gonzáles R,Ballestar JM, Estrada M. A study of the Cuban populations. Red cell and serum biochemical markers. Am J Human Genet 1976;28: 585-596.

5. Sáenz GF, Arroyo G, Jiménez Gutiérrez A. Investigación de hemoglobinas anormales en población de razas negras costarricenses. Rev Biol Trop 1971;19:251-256.

6. Gijzel WP, Adhin SD, Demrawsingh I. Abnormal hemoglobins in Surinan. I. Frequency among the different ethnic groups. Surinan Med Bull 1978;1:18.

7. Pik C, Loos JA, Jonhis JHP, Prins HK. Hereditary and acquired blood factors in the Negroid population of Surinam. II. The incidence of hemoglobin anomalies and deficiency of glucose-6-phosphatedehydrogenase. Trop Geogr Med 1965;17:61-68.

8. Gentilini M, La Roche V, Degremont A. Aspects de la pathologie tropicale parasitaire et infectieuse en Republique de Haití. Bull Soc Path Exot Fil 1964;57:565-570.

9. Restrepo A. Frequency and distribution of abnormal hemoglobins and thalassemias in Colombia. In: Arends T, Brenski G, Nagel R, editors. Genetical, functional and physical studies of hemoglobins. Basel-S-Karker; 1971. p. 52.

10. Tejada C, González NLC, Sánchez M. El factor Diego y el gen de células falciformes entre los caribes de raza negra en Livingstone, Guatemala. Revista Colegio Médico 1965;16:83-5.

11. Nagel RL. The origin of the hemoglobin $S$ gene: clinical, genetic and anthropological consequences. Einstein Quart J Biol Med 1984;2:53-62.

12. Neel JV. Genetics of human hemoglobin differences, problems and perpectives. An Hum Genet 1956;21:1-8.

13. Luzzatti L, Nwachku-Jarrett ES, Reddy S. Increased sickling of parasitized erithrocytes as a mechanism of resistance against malaria in the sickle call trait. Lancet 1970;7642:319-25.

14. Pasvol G. The interaction between sickle hemoglobin and the malaria parasite Plasmodium falciparum. Trans Roy Soc Trop Med Hyg 1980;77:701-5.

15. Guderian RH, Cruz M, Herdoiza VM. Evaluación de los marcadores genéticos asociados con la susceptibilidad genética a la malaria en el Ecuador. En: Memorias. III Congreso Ecuatoriano de Medicina Tropical y Parasitología. Guayaquil: Ed. Semetrop; 1988. p. 481-6.

16. Roth EF. Red cell polymorphisms and the malaria hypothesis. Diagn Med 1985;8:29-35.

17. Yuthavong $\mathbf{Y}$, Wilairat $\mathbf{P}$. Protection against malaria by thalassaemia and haemoglobin variants. Parasitol Today 1993;9:241-5. 